\title{
Minkowski-type inequalities involving Hardy function and symmetric functions
}

\author{
JiaJin Wen ${ }^{1}$, ShanHe Wu ${ }^{2 *}$ and TianYong Han ${ }^{1}$
}

\section{"Correspondence:}

shanhewu@gmail.com

${ }^{2}$ Department of Mathematics and

Computer Science, Longyan

University, Longyan, Fujian 364012,

P.R. China

Full list of author information is

available at the end of the article

\begin{abstract}
The Hardy matrix $H_{n}(\mathbf{x}, \boldsymbol{\alpha})$, the Hardy function per $H_{n}(\mathbf{x}, \boldsymbol{\alpha})$ and the generalized Vandermonde determinant det $H_{n}(\mathbf{x}, \boldsymbol{\alpha})$ are defined in this paper. By means of algebra and analysis theories together with proper hypotheses, we establish the following Minkowski-type inequality involving Hardy function:$$
\left[\operatorname{per} H_{n}(\mathbf{x}+\mathbf{y}, \boldsymbol{\alpha})\right]^{\frac{1}{|\alpha|}} \geq\left[\operatorname{per} H_{n}(\mathbf{x}, \boldsymbol{\alpha})\right]^{\frac{1}{|\alpha|}}+\left[\operatorname{per} H_{n}(\mathbf{y}, \alpha)\right]^{\frac{1}{|\alpha|}} .
$$

As applications, our inequality is used to estimate the lower bounds of the increment of a symmetric function.

MSC: 26D15; 15A15

Keywords: Hardy matrix; Hardy function; Minkowski's inequality; homogeneous and symmetric polynomial; symmetric function
\end{abstract}

\section{Introduction}

We use the following notations throughout the paper (see [1]):

$$
\begin{aligned}
& \mathbf{x} \triangleq\left(x_{1}, \ldots, x_{n}\right), \quad \boldsymbol{\alpha} \triangleq\left(\alpha_{1}, \ldots, \alpha_{n}\right), \quad|\boldsymbol{\alpha}| \triangleq \alpha_{1}+\cdots+\alpha_{n} \\
& \boldsymbol{\varepsilon} \triangleq(0,1, \ldots, n-1), \quad\|\mathbf{x}\|_{p} \triangleq\left(\sum_{i=1}^{n} x_{i}^{p}\right)^{1 / p}, \quad p>0 .
\end{aligned}
$$

Let $A=\left[a_{i, j}\right]_{n \times n}$ be an $n \times n$ matrix over a commutative ring. Then the permanent of the matrix $A$, written as $\operatorname{per} A$, is defined by

$$
\operatorname{per} A \triangleq \sum_{\sigma \in S_{n}} a_{1, \sigma(1)} a_{2, \sigma(2)} \cdots a_{n, \sigma(n)}
$$

where $S_{n}$ is a symmetric group of $n$-order (see [2]). The matrix

$$
H_{n}(\mathbf{x}, \boldsymbol{\alpha}) \triangleq\left[x_{j}^{\alpha_{i}}\right]_{n \times n}=\left[\begin{array}{cccc}
x_{1}^{\alpha_{1}} & x_{2}^{\alpha_{1}} & \ldots & x_{n}^{\alpha_{1}} \\
x_{1}^{\alpha_{2}} & x_{2}^{\alpha_{2}} & \ldots & x_{n}^{\alpha_{2}} \\
\vdots & \vdots & \ddots & \vdots \\
x_{1}^{\alpha_{n}} & x_{2}^{\alpha_{n}} & \cdots & x_{n}^{\alpha_{n}}
\end{array}\right]
$$

○2014 Wen et al.; licensee Springer. This is an Open Access article distributed under the terms of the Creative Commons Attribution License (http://creativecommons.org/licenses/by/2.0), which permits unrestricted use, distribution, and reproduction in any medium, provided the original work is properly cited. 
is called a Hardy matrix, the matrix functions per $H_{n}(\mathbf{x}, \boldsymbol{\alpha})$ and $\operatorname{det} H_{n}(\mathbf{x}, \boldsymbol{\alpha})$ are called the Hardy function (see $[2,3]$ ) and the generalized Vandermonde determinant (see $[4,5]$ ), respectively.

Due to the facts that the symmetric polynomial and certain symmetric functions can be expressed by the Hardy function (see [6] and Remark 1), and that the interpolating quasipolynomial can be expressed by the generalized Vandermonde determinant (see $[4,5])$, the Hardy function and the generalized Vandermonde determinant are of great significance in mathematics.

Obviously, the Hardy function per $H_{n}(\mathbf{x}, \boldsymbol{\alpha})$ is a symmetric function. For the Hardy function, we have the following well-known Hardy inequality (see[3, 7]): Let $\boldsymbol{\alpha}, \boldsymbol{\beta} \in(-\infty, \infty)^{n}$. Then the inequality

$$
\operatorname{per} H_{n}(\mathbf{x}, \boldsymbol{\alpha}) \leq \operatorname{per} H_{n}(\mathbf{x}, \boldsymbol{\beta})
$$

holds for any $\mathbf{x} \in(0, \infty)^{n}$ if and only if $\boldsymbol{\alpha} \prec \boldsymbol{\beta}$.

For the Hardy function per $H_{n}(\mathbf{x}, \boldsymbol{\alpha})$, Wen and Wang in [2] (see Corollary 1 in [2]) obtained the following result: Let $\mathbf{x}, \mathbf{y} \in(0, \infty)^{n}, \boldsymbol{\alpha} \in(-\infty, \infty)^{n}$. If

$$
x_{1} \leq x_{2} \leq \cdots \leq x_{n} \quad \text { and } \quad y_{1} \leq y_{2} \leq \cdots \leq y_{n}
$$

then

$$
\frac{\operatorname{per} H_{n}(\mathbf{x y}, \boldsymbol{\alpha})}{n !} \geq \frac{\operatorname{per} H_{n}(\mathbf{x}, \boldsymbol{\alpha})}{n !} \times \frac{\operatorname{per} H_{n}(\mathbf{y}, \boldsymbol{\alpha})}{n !}
$$

where

$$
\mathbf{x y} \triangleq\left(x_{1} y_{1}, \ldots, x_{n} y_{n}\right) .
$$

For the generalized Vandermonde determinant $\operatorname{det} H_{n}(\mathbf{x}, \boldsymbol{\alpha})$, Wen and Cheng in [5] (see Lemma 3 in [5]) obtained the following result: Let $\mathbf{x}, \boldsymbol{\alpha} \in(0, \infty)^{n}, n \geq 2$. If

$$
x_{1}<x_{2}<\cdots<x_{n}, \quad \alpha_{j+1}-\alpha_{j} \geq 1, \quad j=1,2, \ldots, n-1,
$$

then we have

$$
\operatorname{det} H_{n}(\mathbf{x}, \boldsymbol{\alpha}) \leq\left(\prod_{j=1}^{n-1} j !\right)^{-1} \operatorname{det} H_{n}(\boldsymbol{\alpha}, \boldsymbol{\varepsilon}) \operatorname{det} H_{n}(\mathbf{x}, \boldsymbol{\varepsilon})\left(\frac{x_{n-1}^{d_{n}}+x_{n}^{d_{n}}}{2}\right)^{\frac{|\boldsymbol{\alpha}|-|\boldsymbol{\varepsilon}|}{d_{n}}},
$$

where

$$
d_{n} \triangleq \max \left\{1, \alpha_{n}-\alpha_{n-1}-1\right\}, \quad|\boldsymbol{\alpha}|>|\boldsymbol{\varepsilon}|=\frac{n(n-1)}{2} .
$$

Famous Minkowski's inequality can be described as follows (see [8, 9]): If $0<p<1$, then for any $\mathbf{x}, \mathbf{y} \in(0, \infty)^{n}$, we have the inequality

$$
\|\mathbf{x}+\mathbf{y}\|_{p} \geq\|\mathbf{x}\|_{p}+\|\mathbf{y}\|_{p} .
$$


Inequality (4) is reversed if $p>1$. Equality in (4) holds if and only if $\mathbf{x}, \mathbf{y}$ are linearly dependent.

Minkowski's inequality has a wide range of applications, especially in the algebraic geometry and space science (see [8-11]). In this paper, we establish the following Minkowskitype inequality (5) involving Hardy function.

Theorem 1 (Minkowski-type inequality) Let $\boldsymbol{\alpha} \in[0,1]^{n}$. If $0<|\boldsymbol{\alpha}| \leq 1$, then for any $\mathbf{x}, \mathbf{y} \in$ $(0, \infty)^{n}$, we have the following inequality:

$$
\left[\operatorname{per} H_{n}(\mathbf{x}+\mathbf{y}, \boldsymbol{\alpha})\right]^{\frac{1}{|\alpha|}} \geq\left[\operatorname{per} H_{n}(\mathbf{x}, \boldsymbol{\alpha})\right]^{\frac{1}{|\alpha|}}+\left[\operatorname{per} H_{n}(\mathbf{y}, \boldsymbol{\alpha})\right]^{\frac{1}{|\alpha|}} .
$$

Equality in (5) holds if $\mathbf{x}, \mathbf{y}$ are linearly dependent.

In Section 3, we demonstrate the applications of Theorem 1. Our objective is to estimate the lower bounds of the increment of a symmetric function.

\section{The proof of Theorem 1}

In order to prove Theorem 1, we need the following lemmas.

Lemma 1 If $\boldsymbol{\alpha} \in[0, \infty)^{2},|\boldsymbol{\alpha}|-\left(\alpha_{1}-\alpha_{2}\right)^{2} \geq 0,|\boldsymbol{\alpha}|>0$, then for any $\mathbf{x}, \mathbf{y} \in(0, \infty)^{2}$, we have the following Minkowski-type inequality:

$$
\left[\operatorname{per} H_{2}(\mathbf{x}+\mathbf{y}, \boldsymbol{\alpha})\right]^{\frac{1}{|\boldsymbol{\alpha}|}} \geq\left[\operatorname{per} H_{2}(\mathbf{x}, \boldsymbol{\alpha})\right]^{\frac{1}{|\alpha|}}+\left[\operatorname{per} H_{2}(\mathbf{y}, \boldsymbol{\alpha})\right]^{\frac{1}{|\alpha|}} .
$$

Equality in (6) holds if $\mathbf{x}, \mathbf{y}$ are linearly dependent.

Proof First of all, we consider the case

$$
\boldsymbol{\alpha} \in(0, \infty)^{2}, \quad|\boldsymbol{\alpha}|-\left(\alpha_{1}-\alpha_{2}\right)^{2}>0 .
$$

Write $y_{i} / x_{i}=u_{i}, i=1,2$. Then inequality (6) can be rewritten as

$$
\begin{aligned}
& {\left[x_{1}^{\alpha_{1}} x_{2}^{\alpha_{2}}\left(1+u_{1}\right)^{\alpha_{1}}\left(1+u_{2}\right)^{\alpha_{2}}+x_{1}^{\alpha_{2}} x_{2}^{\alpha_{1}}\left(1+u_{1}\right)^{\alpha_{2}}\left(1+u_{2}\right)^{\alpha_{1}}\right]^{\frac{1}{\alpha_{1}+\alpha_{2}}}} \\
& \quad \geq\left(x_{1}^{\alpha_{1}} x_{2}^{\alpha_{2}} u_{1}^{\alpha_{1}} u_{2}^{\alpha_{2}}+x_{1}^{\alpha_{2}} x_{2}^{\alpha_{1}} u_{1}^{\alpha_{2}} u_{2}^{\alpha_{1}}\right)^{\frac{1}{\alpha_{1}+\alpha_{2}}}+\left(x_{1}^{\alpha_{1}} x_{2}^{\alpha_{2}}+x_{1}^{\alpha_{2}} x_{2}^{\alpha_{1}}\right)^{\frac{1}{\alpha_{1}+\alpha_{2}}} .
\end{aligned}
$$

Without loss of generality, we can assume that

$$
x_{1}^{\alpha_{1}} x_{2}^{\alpha_{2}}+x_{1}^{\alpha_{2}} x_{2}^{\alpha_{1}}=1, \quad\left(x_{1}, x_{2}\right) \in(0, \infty)^{2} .
$$

Indeed, if

$$
x_{1}^{\alpha_{1}} x_{2}^{\alpha_{2}}+x_{1}^{\alpha_{2}} x_{2}^{\alpha_{1}}=C^{\alpha_{1}+\alpha_{2}}, \quad C>0,
$$

then

$$
x_{1}^{* \alpha_{1}} x_{2}^{* \alpha_{2}}+x_{1}^{* \alpha_{2}} x_{2}^{* \alpha_{1}}=1, \quad\left(x_{1}^{*}, x_{2}^{*}\right) \in(0, \infty)^{2},
$$


where

$$
x_{i}^{*}=C^{-1} x_{i}, \quad i=1,2 .
$$

Set

$$
x_{1}^{\alpha_{1}} x_{2}^{\alpha_{2}} u_{1}^{\alpha_{1}} u_{2}^{\alpha_{2}}+x_{1}^{\alpha_{2}} x_{2}^{\alpha_{1}} u_{1}^{\alpha_{2}} u_{2}^{\alpha_{1}}=c^{\alpha_{1}+\alpha_{2}}, \quad c>0,
$$

and

$$
\begin{aligned}
& F\left(u_{1}, u_{2}\right) \triangleq x_{1}^{\alpha_{1}} x_{2}^{\alpha_{2}}\left(1+u_{1}\right)^{\alpha_{1}}\left(1+u_{2}\right)^{\alpha_{2}}+x_{1}^{\alpha_{2}} x_{2}^{\alpha_{1}}\left(1+u_{1}\right)^{\alpha_{2}}\left(1+u_{2}\right)^{\alpha_{1}}, \\
& D \triangleq\left\{\left(u_{1}, u_{2}\right) \in(0, \infty)^{2} \mid x_{1}^{\alpha_{1}} x_{2}^{\alpha_{2}} u_{1}^{\alpha_{1}} u_{2}^{\alpha_{2}}+x_{1}^{\alpha_{2}} x_{2}^{\alpha_{1}} u_{1}^{\alpha_{2}} u_{2}^{\alpha_{1}}=c^{\alpha_{1}+\alpha_{2}}\right\} .
\end{aligned}
$$

We arbitrarily fixed $x_{1}, x_{2}$, which satisfies condition (8), then inequality (6) can be rewritten as

$$
F\left(u_{1}, u_{2}\right) \geq(c+1)^{\alpha_{1}+\alpha_{2}}, \quad \forall\left(u_{1}, u_{2}\right) \in D .
$$

We consider the following Lagrange function:

$$
L=F\left(u_{1}, u_{2}\right)+\lambda\left(x_{1}^{\alpha_{1}} x_{2}^{\alpha_{2}} u_{1}^{\alpha_{1}} u_{2}^{\alpha_{2}}+x_{1}^{\alpha_{2}} x_{2}^{\alpha_{1}} u_{1}^{\alpha_{2}} u_{2}^{\alpha_{1}}-c^{\alpha_{1}+\alpha_{2}}\right) .
$$

Set

$$
\begin{aligned}
\frac{\partial L}{\partial u_{1}}= & \alpha_{1} x_{1}^{\alpha_{1}} x_{2}^{\alpha_{2}}\left(1+u_{1}\right)^{\alpha_{1}-1}\left(1+u_{2}\right)^{\alpha_{2}}+\alpha_{2} x_{1}^{\alpha_{2}} x_{2}^{\alpha_{1}}\left(1+u_{1}\right)^{\alpha_{2}-1}\left(1+u_{2}\right)^{\alpha_{1}} \\
& +\lambda\left(\alpha_{1} x_{1}^{\alpha_{1}} x_{2}^{\alpha_{2}} u_{1}^{\alpha_{1}-1} u_{2}^{\alpha_{2}}+\alpha_{2} x_{1}^{\alpha_{2}} x_{2}^{\alpha_{1}} u_{1}^{\alpha_{2}-1} u_{2}^{\alpha_{1}}\right)=0,
\end{aligned}
$$

and

$$
\begin{aligned}
\frac{\partial L}{\partial u_{2}}= & \alpha_{2} x_{1}^{\alpha_{1}} x_{2}^{\alpha_{2}}\left(1+u_{1}\right)^{\alpha_{1}}\left(1+u_{2}\right)^{\alpha_{2}-1}+\alpha_{1} x_{1}^{\alpha_{2}} x_{2}^{\alpha_{1}}\left(1+u_{1}\right)^{\alpha_{2}}\left(1+u_{2}\right)^{\alpha_{1}-1} \\
& +\lambda\left(\alpha_{2} x_{1}^{\alpha_{1}} x_{2}^{\alpha_{2}} u_{1}^{\alpha_{1}} u_{2}^{\alpha_{2}-1}+\alpha_{1} x_{1}^{\alpha_{2}} x_{2}^{\alpha_{1}} u_{1}^{\alpha_{2}} u_{2}^{\alpha_{1}-1}\right)=0 .
\end{aligned}
$$

From (11) and (12), we get

$$
\begin{aligned}
& \frac{\alpha_{1} x_{1}^{\alpha_{1}} x_{2}^{\alpha_{2}}\left(1+u_{1}\right)^{\alpha_{1}-1}\left(1+u_{2}\right)^{\alpha_{2}}+\alpha_{2} x_{1}^{\alpha_{2}} x_{2}^{\alpha_{1}}\left(1+u_{1}\right)^{\alpha_{2}-1}\left(1+u_{2}\right)^{\alpha_{1}}}{\alpha_{2} x_{1}^{\alpha_{1}} x_{2}^{\alpha_{2}}\left(1+u_{1}\right)^{\alpha_{1}}\left(1+u_{2}\right)^{\alpha_{2}-1}+\alpha_{1} x_{1}^{\alpha_{2}} x_{2}^{\alpha_{1}}\left(1+u_{1}\right)^{\alpha_{2}}\left(1+u_{2}\right)^{\alpha_{1}-1}} \\
& \quad=\frac{\alpha_{1} x_{1}^{\alpha_{1}} x_{2}^{\alpha_{2}} u_{1}^{\alpha_{1}-1} u_{2}^{\alpha_{2}}+\alpha_{2} x_{1}^{\alpha_{2}} x_{2}^{\alpha_{1}} u_{1}^{\alpha_{2}-1} u_{2}^{\alpha_{1}}}{\alpha_{2} x_{1}^{\alpha_{1}} x_{2}^{\alpha_{2}} u_{1}^{\alpha_{1}} u_{2}^{\alpha_{2}-1}+\alpha_{1} x_{1}^{\alpha_{2}} x_{2}^{\alpha_{1}} u_{1}^{\alpha_{2}} u_{2}^{\alpha_{1}-1}} .
\end{aligned}
$$

Write

$$
\mu \triangleq\left(\frac{x_{2}}{x_{1}}\right)^{\alpha_{1}-\alpha_{2}}>0, \quad g(t) \triangleq t \frac{\alpha_{1}+\mu \alpha_{2} t^{\alpha_{1}-\alpha_{2}}}{\alpha_{2}+\mu \alpha_{1} t^{\alpha_{1}-\alpha_{2}}}, \quad t>0 .
$$


Since

$$
\frac{\alpha_{1} x_{1}^{\alpha_{1}} x_{2}^{\alpha_{2}} u_{1}^{\alpha_{1}-1} u_{2}^{\alpha_{2}}+\alpha_{2} x_{1}^{\alpha_{2}} x_{2}^{\alpha_{1}} u_{1}^{\alpha_{2}-1} u_{2}^{\alpha_{1}}}{\alpha_{2} x_{1}^{\alpha_{1}} x_{2}^{\alpha_{2}} u_{1}^{\alpha_{1}} u_{2}^{\alpha_{2}-1}+\alpha_{1} x_{1}^{\alpha_{2}} x_{2}^{\alpha_{1}} u_{1}^{\alpha_{2}} u_{2}^{\alpha_{1}-1}}=\frac{u_{2}}{u_{1}} \frac{\alpha_{1}+\alpha_{2}\left(\frac{x_{2}}{x_{1}}\right)^{\alpha_{1}-\alpha_{2}}\left(\frac{u_{2}}{u_{1}}\right)^{\alpha_{1}-\alpha_{2}}}{\alpha_{2}+\alpha_{1}\left(\frac{x_{2}}{x_{1}}\right)^{\alpha_{1}-\alpha_{2}}\left(\frac{u_{2}}{u_{1}}\right)^{\alpha_{1}-\alpha_{2}}},
$$

equation (13) can be rewritten as

$$
g\left(\frac{1+u_{2}}{1+u_{1}}\right)=g\left(\frac{u_{2}}{u_{1}}\right)
$$

By

$$
\log [g(t)]=\log (t)+\log \left(\alpha_{1}+\mu \alpha_{2} t^{\alpha_{1}-\alpha_{2}}\right)-\log \left(\alpha_{2}+\mu \alpha_{1} t^{\alpha_{1}-\alpha_{2}}\right)
$$

we get

$$
\begin{aligned}
\frac{g^{\prime}(t)}{g(t)} & =\frac{1}{t}+\frac{\left(\alpha_{1}-\alpha_{2}\right) \mu \alpha_{2} t^{\alpha_{1}-\alpha_{2}-1}}{\alpha_{1}+\mu \alpha_{2} t^{\alpha_{1}-\alpha_{2}}}-\frac{\left(\alpha_{1}-\alpha_{2}\right) \mu \alpha_{1} t^{\alpha_{1}-\alpha_{2}-1}}{\alpha_{2}+\mu \alpha_{1} t^{\alpha_{1}-\alpha_{2}}} \\
& =\frac{\alpha_{1} \alpha_{2}\left[1+\left(\mu t^{\alpha_{1}-\alpha_{2}}\right)^{2}\right]+\left[\alpha_{1}^{2}+\alpha_{2}^{2}+\left(\alpha_{1}-\alpha_{2}\right)\left(\alpha_{2}^{2}-\alpha_{1}^{2}\right)\right] \mu t^{\alpha_{1}-\alpha_{2}}}{t\left(\alpha_{1}+\mu \alpha_{2} t^{\alpha_{1}-\alpha_{2}}\right)\left(\alpha_{2}+\mu \alpha_{1} t^{\alpha_{1}-\alpha_{2}}\right)} \\
& \geq \frac{2 \alpha_{1} \alpha_{2} \mu t^{\alpha_{1}-\alpha_{2}}+\left[\alpha_{1}^{2}+\alpha_{2}^{2}+\left(\alpha_{1}-\alpha_{2}\right)\left(\alpha_{2}^{2}-\alpha_{1}^{2}\right)\right] \mu t^{\alpha_{1}-\alpha_{2}}}{t\left(\alpha_{1}+\mu \alpha_{2} t^{\alpha_{1}-\alpha_{2}}\right)\left(\alpha_{2}+\mu \alpha_{1} t^{\alpha_{1}-\alpha_{2}}\right)} \\
& =\frac{\left(\alpha_{1}+\alpha_{2}\right)\left[\alpha_{1}+\alpha_{2}-\left(\alpha_{1}-\alpha_{2}\right)^{2}\right]}{t\left(\alpha_{1}+\mu \alpha_{2} t^{\alpha_{1}-\alpha_{2}}\right)\left(\alpha_{2}+\mu \alpha_{1} t^{\alpha_{1}-\alpha_{2}}\right)} \mu t^{\alpha_{1}-\alpha_{2}} \\
& >0
\end{aligned}
$$

hence

$$
g^{\prime}(t)>0, \quad \forall t>0
$$

By (15) and (16), we get

$$
\frac{1+u_{2}}{1+u_{1}}=\frac{u_{2}}{u_{1}}
$$

By (16), (17), (8) and (9), we get

$$
u_{1}=u_{2}=c \text {. }
$$

According to the theory of mathematical analysis, we just need to prove that inequality (10) holds for a stationary point $(c, c)$ of $F\left(u_{1}, u_{2}\right)$ and boundary points of $D$.

If $\left(u_{1}, u_{2}\right)=(c, c) \in D$ is a stationary point of $F\left(u_{1}, u_{2}\right)$, then equality in (10) holds. Here we assume that $\left(u_{1}, u_{2}\right)$ is a boundary point of $D$. Then we have $\left(u_{1}, u_{2}\right)=(0, \infty)$ or $\left(u_{1}, u_{2}\right)=$ $(\infty, 0)$. Since

$$
F\left(u_{1}, u_{2}\right)=\infty>(c+1)^{\alpha_{1}+\alpha_{2}},
$$

inequality (10) also holds. So we have proved inequalities (7) and (6). 
Next, note the continuity of both sides of (6) for the variable $\boldsymbol{\alpha}$, hence inequality (6) also holds if

$$
\boldsymbol{\alpha} \in[0, \infty)^{2}, \quad|\boldsymbol{\alpha}|-\left(\alpha_{1}-\alpha_{2}\right)^{2}=0, \quad|\boldsymbol{\alpha}|>0
$$

From the above analysis we know that equality in (6) holds if $u_{1}=u_{2}$, i.e., $\mathbf{x}, \mathbf{y}$ are linearly dependent. This completes the proof of Lemma 1.

Lemma 2 If $\boldsymbol{\alpha} \in(0,1)^{2}$ and $0<|\boldsymbol{\alpha}|<1$, then for any $\mathbf{x}, \mathbf{y}, \mathbf{z}, \mathbf{w} \in(0, \infty)^{n}, n \geq 1$, we have the inequality

$$
\left[\sum_{i=1}^{n}\left(x_{i}+y_{i}\right)^{\alpha_{1}}\left(z_{i}+w_{i}\right)^{\alpha_{2}}\right]^{\frac{1}{|\boldsymbol{\alpha}|}} \geq\left(\sum_{i=1}^{n} x_{i}^{\alpha_{1}} z_{i}^{\alpha_{2}}\right)^{\frac{1}{|\boldsymbol{\alpha}|}}+\left(\sum_{i=1}^{n} y_{i}^{\alpha_{1}} w_{i}^{\alpha_{2}}\right)^{\frac{1}{|\boldsymbol{\alpha}|}} .
$$

Equation in (19) holds if and only if

$$
\frac{x_{1}}{y_{1}}=\frac{x_{2}}{y_{2}}=\cdots=\frac{x_{n}}{y_{n}}=\frac{z_{1}}{w_{1}}=\frac{z_{2}}{w_{2}}=\cdots=\frac{z_{n}}{w_{n}} .
$$

Proof Write

$$
\frac{y_{i}}{x_{i}}=u_{i}, \quad \frac{w_{i}}{z_{i}}=v_{i}, \quad i=1,2 .
$$

Then inequality (19) can be rewritten as

$$
\left[\sum_{i=1}^{n} x_{i}^{\alpha_{1}} z_{i}^{\alpha_{2}}\left(1+u_{i}\right)^{\alpha_{1}}\left(1+v_{i}\right)^{\alpha_{2}}\right]^{\frac{1}{|\alpha|}} \geq\left(\sum_{i=1}^{n} x_{i}^{\alpha_{1}} z_{i}^{\alpha_{2}} u_{i}^{\alpha_{1}} v_{i}^{\alpha_{2}}\right)^{\frac{1}{|\boldsymbol{\alpha}|}}+\left(\sum_{i=1}^{n} x_{i}^{\alpha_{1}} z_{i}^{\alpha_{2}}\right)^{\frac{1}{|\boldsymbol{\alpha}|}}
$$

Without loss of generality, we can assume that

$$
\sum_{i=1}^{n} x_{i}^{\alpha_{1}} z_{i}^{\alpha_{2}}=1, \quad \mathbf{x}, \mathbf{z} \in(0, \infty)^{n}
$$

and

$$
\sum_{i=1}^{n} x_{i}^{\alpha_{1}} z_{i}^{\alpha_{2}} u_{i}^{\alpha_{1}} v_{i}^{\alpha_{2}}=c^{\alpha_{1}+\alpha_{2}}, \quad c>0
$$

Write

$$
G(u, v) \triangleq \sum_{i=1}^{n} x_{i}^{\alpha_{1}} z_{i}^{\alpha_{2}}\left(1+u_{i}\right)^{\alpha_{1}}\left(1+v_{i}\right)^{\alpha_{2}}, \quad u, v \in(0, \infty)^{n}
$$

and

$$
D_{*} \triangleq\left\{(u, v) \in(0, \infty)^{2 n} \mid \sum_{i=1}^{n} x_{i}^{\alpha_{1}} z_{i}^{\alpha_{2}} u_{i}^{\alpha_{1}} v_{i}^{\alpha_{2}}=c^{\alpha_{1}+\alpha_{2}}\right\} .
$$


Then inequality (21) can be rewritten as

$$
G(u, v) \geq(c+1)^{\alpha_{1}+\alpha_{2}}, \quad \forall(u, v) \in D_{*} .
$$

We define the following Lagrange function:

$$
L=G(u, v)+\lambda\left(\sum_{i=1}^{n} x_{i}^{\alpha_{1}} z_{i}^{\alpha_{2}} u_{i}^{\alpha_{1}} v_{i}^{\alpha_{2}}-c^{\alpha_{1}+\alpha_{2}}\right)
$$

Set

$$
\frac{\partial L}{\partial u_{k}}=\alpha_{1} x_{k}^{\alpha_{1}} z_{k}^{\alpha_{2}}\left(1+u_{k}\right)^{\alpha_{1}-1}\left(1+v_{k}\right)^{\alpha_{2}}+\lambda \alpha_{1} x_{k}^{\alpha_{1}} z_{k}^{\alpha_{2}} u_{k}^{\alpha_{1}-1} v_{k}^{\alpha_{2}}=0, \quad k=1,2, \ldots, n,
$$

and

$$
\frac{\partial L}{\partial v_{k}}=\alpha_{2} x_{k}^{\alpha_{1}} z_{k}^{\alpha_{2}}\left(1+u_{k}\right)^{\alpha_{1}}\left(1+v_{k}\right)^{\alpha_{2}-1}+\lambda \alpha_{2} x_{k}^{\alpha_{1}} z_{k}^{\alpha_{2}} u_{k}^{\alpha_{1}} v_{k}^{\alpha_{1}-1}=0, \quad k=1,2, \ldots, n
$$

Then equations (25) and (26) can be rewritten as

$$
\alpha_{1} x_{k}^{\alpha_{1}} z_{k}^{\alpha_{2}}\left(1+u_{k}\right)^{\alpha_{1}-1}\left(1+v_{k}\right)^{\alpha_{2}}=-\lambda \alpha_{1} x_{k}^{\alpha_{1}} z_{k}^{\alpha_{2}} u_{k}^{\alpha_{1}-1} v_{k}^{\alpha_{2}}, \quad k=1,2, \ldots, n,
$$

and

$$
\alpha_{2} x_{k}^{\alpha_{1}} z_{k}^{\alpha_{2}}\left(1+u_{k}\right)^{\alpha_{1}}\left(1+v_{k}\right)^{\alpha_{2}-1}=-\lambda \alpha_{2} x_{k}^{\alpha_{1}} z_{k}^{\alpha_{2}} u_{k}^{\alpha_{1}} v_{k}^{\alpha_{1}-1}, \quad k=1,2, \ldots, n,
$$

respectively. From (27) divided by (28), we get

$$
\frac{1+v_{k}}{1+u_{k}}=\frac{v_{k}}{u_{k}} \Leftrightarrow u_{k}=v_{k}, \quad k=1,2, \ldots, n .
$$

From (29) and (27), we get

$$
\left(1+u_{k}^{-1}\right)^{\alpha_{1}+\alpha_{2}-1}=-\lambda, \quad k=1,2, \ldots, n .
$$

By (30) and $\alpha_{1}+\alpha_{2}-1=|\boldsymbol{\alpha}|-1<0$, we get

$$
u_{1}=u_{2}=\cdots=u_{n}
$$

From (31), (29), (22) and (23), we get

$$
u_{1}=u_{2}=\cdots=u_{n}=v_{1}=v_{2}=\cdots=v_{n}=c .
$$

That is to say, the function $G(u, v)$ has a unique stationary point $(c, \ldots, c, c, \ldots, c)$ in $D_{*}$.

Next, we use the mathematical induction to prove that inequality (24) holds as follows. According to the theory of mathematical analysis, we only need to prove that inequality (24) holds for a stationary point $(c, \ldots, c, c, \ldots, c)$ of $G(u, v)$ and boundary points of $D_{*}$. To complete our proof, we need to divide it into two steps (A) and (B). 
(A) Let $n=1$. If $(c, c)$ is a stationary point of $G(u, v)$ in $D_{*}$, then equality in (24) holds. Here we assume that $(u, v)$ is a boundary point of $D_{*}$. From $(23)$ we know that $\left(u_{1}, v_{1}\right)=(0, \infty)$ or $\left(u_{1}, v_{1}\right)=(\infty, 0)$. Hence

$$
G(u, v)=x_{1}^{\alpha_{1}} z_{1}^{\alpha_{2}}\left(1+u_{1}\right)^{\alpha_{1}}\left(1+v_{1}\right)^{\alpha_{2}}=\infty>c^{\alpha_{1}+\alpha_{2}} .
$$

That is to say, inequality (24) also holds. According to the theory of mathematical analysis, inequality (24) is proved.

Let $n=2$. If $(c, c, c, c)$ is a stationary point of $G(u, v)$ in $D_{*}$, then equality in (24) holds. Here we assume that $(u, v)$ is a boundary point of $D_{*}$, then there is a 0 among $u_{1}, u_{2}, v_{1}$, $v_{2}$. Without loss of generality, we can assume that $u_{2}=0$. From (23) we have

$$
x_{1}^{\alpha_{1}} z_{1}^{\alpha_{2}} u_{1}^{\alpha_{1}} v_{1}^{\alpha_{2}}=c^{\alpha_{1}+\alpha_{2}}, \quad c>0 .
$$

By (33), we get

$$
\begin{aligned}
G(u, v) & =x_{1}^{\alpha_{1}} z_{1}^{\alpha_{2}}\left(1+u_{1}\right)^{\alpha_{1}}\left(1+v_{1}\right)^{\alpha_{2}}+x_{2}^{\alpha_{1}} z_{2}^{\alpha_{2}}\left(1+u_{2}\right)^{\alpha_{1}}\left(1+v_{2}\right)^{\alpha_{2}} \\
& =x_{1}^{\alpha_{1}} z_{1}^{\alpha_{2}}\left(1+u_{1}\right)^{\alpha_{1}}\left(1+v_{1}\right)^{\alpha_{2}}+x_{2}^{\alpha_{1}} z_{2}^{\alpha_{2}}\left(1+v_{2}\right)^{\alpha_{2}} \\
& >x_{1}^{\alpha_{1}} z_{1}^{\alpha_{2}}\left(1+u_{1}\right)^{\alpha_{1}}\left(1+v_{1}\right)^{\alpha_{2}} \\
& >x_{1}^{\alpha_{1}} z_{1}^{\alpha_{2}} u_{1}^{\alpha_{1}} v_{1}^{\alpha_{2}} \\
& =c^{\alpha_{1}+\alpha_{2}} .
\end{aligned}
$$

That is to say, inequality (24) still holds for the case when $(u, v)$ is a boundary point of $D_{*}$. According to the theory of mathematical analysis, we know that inequality (24) is proved.

(B) Suppose that inequality (24) holds if we use $n-1(n \geq 3)$ instead of $n$, we prove that inequality (24) holds as follows.

For a stationary point $(c, \ldots, c, c, \ldots, c)$ of $G(u, v)$ in $D_{*}$, equation in (24) holds. Here we assume that $(u, v)$ is a boundary point of $D_{*}$, then there is a 0 among $u_{1}, u_{2}, \ldots, u_{n}$, $v_{1}, v_{2}, \ldots, v_{n}$. Without loss of generality, we can assume that $u_{n}=0$. From (23), we get

$$
\sum_{i=1}^{n-1} x_{i}^{\alpha_{1}} z_{i}^{\alpha_{2}} u_{i}^{\alpha_{1}} v_{i}^{\alpha_{2}}=c^{\alpha_{1}+\alpha_{2}}, \quad c>0
$$

Set

$$
x_{i}^{*}=x_{i}\left(1-x_{n}^{\alpha_{1}} z_{n}^{\alpha_{2}}\right)^{-\frac{1}{\alpha_{1}+\alpha_{2}}}, \quad z_{i}^{*}=z_{i}\left(1-x_{n}^{\alpha_{1}} z_{n}^{\alpha_{2}}\right)^{-\frac{1}{\alpha_{1}+\alpha_{2}}}, \quad i=1,2, \ldots, n-1,
$$

then equation (22) can be rewritten as

$$
\sum_{i=1}^{n-1} x_{i}^{* \alpha_{1}} z_{i}^{* \alpha_{2}}=1, \quad x^{*}, z^{*} \in(0, \infty)^{n-1}
$$

and equation (23) can be rewritten as

$$
\sum_{i=1}^{n-1} x_{i}^{* \alpha_{1}} z_{i}^{* \alpha_{2}} u_{i}^{\alpha_{1}} v_{i}^{\alpha_{2}}=c_{*}^{\alpha_{1}+\alpha_{2}}, \quad c_{*}=c\left(1-x_{n}^{\alpha_{1}} z_{n}^{\alpha_{2}}\right)^{-\frac{1}{\alpha_{1}+\alpha_{2}}}>0
$$


as well as the function $G(u, v)$ can be rewritten as

$$
G(u, v)=\left(1-x_{n}^{\alpha_{1}} z_{n}^{\alpha_{2}}\right) \sum_{i=1}^{n-1} x_{i}^{* \alpha_{1}} z_{i}^{* \alpha_{2}}\left(1+u_{i}\right)^{\alpha_{1}}\left(1+v_{i}\right)^{\alpha_{2}}+x_{n}^{\alpha_{1}} z_{n}^{\alpha_{2}}\left(1+v_{n}\right)^{\alpha_{2}} .
$$

By the induction hypothesis we have

$$
\sum_{i=1}^{n-1} x_{i}^{* \alpha_{1}} z_{i}^{* \alpha_{2}}\left(1+u_{i}\right)^{\alpha_{1}}\left(1+v_{i}\right)^{\alpha_{2}} \geq\left(c_{*}+1\right)^{\alpha_{1}+\alpha_{2}} .
$$

By (37) and (38), we get

$$
\begin{aligned}
G(u, v) & =\left(1-x_{n}^{\alpha_{1}} z_{n}^{\alpha_{2}}\right) \sum_{i=1}^{n-1} x_{i}^{* \alpha_{1}} z_{i}^{* \alpha_{2}}\left(1+u_{i}\right)^{\alpha_{1}}\left(1+v_{i}\right)^{\alpha_{2}}+x_{n}^{\alpha_{1}} z_{n}^{\alpha_{2}}\left(1+v_{n}\right)^{\alpha_{2}} \\
& \geq\left(1-x_{n}^{\alpha_{1}} z_{n}^{\alpha_{2}}\right) \sum_{i=1}^{n-1} x_{i}^{* \alpha_{1}} z_{i}^{* \alpha_{2}}\left(1+u_{i}\right)^{\alpha_{1}}\left(1+v_{i}\right)^{\alpha_{2}}+x_{n}^{\alpha_{1}} z_{n}^{\alpha_{2}} \\
& \geq\left(1-x_{n}^{\alpha_{1}} z_{n}^{\alpha_{2}}\right)\left(c_{*}+1\right)^{\alpha_{1}+\alpha_{2}}+x_{n}^{\alpha_{1}} z_{n}^{\alpha_{2}} \\
& =\left(1-x_{n}^{\alpha_{1}} z_{n}^{\alpha_{2}}\right)\left[c\left(1-x_{n}^{\alpha_{1}} z_{n}^{\alpha_{2}}\right)^{-\frac{1}{\alpha_{1}+\alpha_{2}}}+1\right]^{\alpha_{1}+\alpha_{2}}+x_{n}^{\alpha_{1}} z_{n}^{\alpha_{2}} \\
& =\left[c+\left(1-x_{n}^{\alpha_{1}} z_{n}^{\alpha_{2}}\right)^{\frac{1}{\alpha_{1}+\alpha_{2}}}\right]^{\alpha_{1}+\alpha_{2}}+x_{n}^{\alpha_{1}} z_{n}^{\alpha_{2}},
\end{aligned}
$$

i.e.,

$$
G(u, v) \geq \varphi(\delta)
$$

where

$$
\delta \triangleq\left(1-x_{n}^{\alpha_{1}} z_{n}^{\alpha_{2}}\right)^{\frac{1}{\alpha_{1}+\alpha_{2}}} \in(0,1), \quad x_{n}^{\alpha_{1}} z_{n}^{\alpha_{2}}=1-\delta^{\alpha_{1}+\alpha_{2}},
$$

and

$$
\varphi(\delta) \triangleq(c+\delta)^{\alpha_{1}+\alpha_{2}}+1-\delta^{\alpha_{1}+\alpha_{2}}, \quad \delta \in(0,1) .
$$

Since

$$
\alpha_{1}+\alpha_{2}>0, \quad \alpha_{1}+\alpha_{2}-1=|\alpha|-1<0, \quad c>0,
$$

we have

$$
\frac{d \varphi(\delta)}{d \delta} \triangleq\left(\alpha_{1}+\alpha_{2}\right)\left[(c+\delta)^{\alpha_{1}+\alpha_{2}-1}-\delta^{\alpha_{1}+\alpha_{2}-1}\right]<0, \quad \delta \in(0,1) .
$$

From $\delta \in(0,1)$ and $(41)$, we get

$$
\varphi(\delta) \triangleq(c+\delta)^{\alpha_{1}+\alpha_{2}}+1-\delta^{\alpha_{1}+\alpha_{2}}>\varphi(1)=(c+1)^{\alpha_{1}+\alpha_{2}} .
$$


Combining with inequalities (39) and (42), we get

$$
G(u, v) \geq \varphi(\delta)>(c+1)^{\alpha_{1}+\alpha_{2}} .
$$

By inequality (43) we know that inequality (24) holds.

According to the theory of mathematical analysis, we know that inequality (24) is proved, hence inequality (19) is also proved by the above analysis. Inequality (19) is an equation if and only if equations (20) hold. This completes the proof of Lemma 2.

Next we turn to the proof of Theorem 1.

Proof First of all, we prove that inequality (5) holds if $\boldsymbol{\alpha} \in(0,1)^{n}$ and $0<|\boldsymbol{\alpha}|<1$ by induction for $n$. To complete our proof, we need to divide it into two steps (A) and (B).

(A) When $n=1$, then inequality (5) is an equation. Let $n=2$. According to the hypothesis of Theorem 1, we know that

$$
\left|\alpha_{1}-\alpha_{2}\right|<\left|\alpha_{1}+\alpha_{2}\right|<1, \quad \alpha_{1}+\alpha_{2}-\left(\alpha_{1}-\alpha_{2}\right)^{2}>\alpha_{1}+\alpha_{2}-\left(\alpha_{1}+\alpha_{2}\right)^{2}>0 .
$$

By Lemma 1, inequality (5) holds.

(B) Suppose that inequality (5) holds if we use $n-1(n \geq 3)$ instead of $n$, we prove that inequality (5) holds as follows.

For convenience, we use the following notations:

$$
\mathbf{x}(j)=\left(x_{1}, \ldots, x_{j-1}, x_{j+1}, \ldots, x_{n}\right), \quad \boldsymbol{\alpha}(n)=\left(\alpha_{1}, \alpha_{2}, \ldots, \alpha_{n-1}\right) .
$$

According to the Laplace theorem (see [2]), we obtain that

$$
\operatorname{per} H_{n}(\mathbf{x}, \boldsymbol{\alpha})=\sum_{j=1}^{n} x_{j}^{\alpha_{n}} \operatorname{per} H_{n-1}(\mathbf{x}(j), \boldsymbol{\alpha}(n))
$$

Write

$$
z_{j} \triangleq\left(\operatorname{per} H_{n-1}(\mathbf{x}(j), \boldsymbol{\alpha}(n))\right)^{\frac{1}{|\alpha(n)|}} \quad \text { and } \quad w_{j} \triangleq\left(\operatorname{per} H_{n-1}(\mathbf{y}(j), \boldsymbol{\alpha}(n))\right)^{\frac{1}{|\alpha(n)|}} \text {. }
$$

By

$$
0<|\boldsymbol{\alpha}(n)|<\alpha_{n}+|\boldsymbol{\alpha}(n)|=|\boldsymbol{\alpha}|<1,
$$

(44), the induction hypothesis and Lemma 2, we get

$$
\begin{aligned}
& {\left[\operatorname{per} H_{n}(\mathbf{x}+\mathbf{y}, \boldsymbol{\alpha})\right]^{\frac{1}{|\alpha|}}} \\
& \quad=\left\{\sum_{j=1}^{n}\left(x_{j}+y_{j}\right)^{\alpha_{n}}\left[\operatorname{per} H_{n-1}(\mathbf{x}(j)+\mathbf{y}(j), \boldsymbol{\alpha}(n))\right]^{\frac{|\alpha(n)|}{|\alpha(n)|}}\right\}^{\frac{1}{|\boldsymbol{\alpha}|}} \\
& \geq\left[\sum_{j=1}^{n}\left(x_{j}+y_{j}\right)^{\alpha_{n}}\left(z_{j}+w_{j}\right)^{|\boldsymbol{\alpha}(n)|}\right]^{\frac{1}{|\boldsymbol{\alpha}|}}
\end{aligned}
$$




$$
\begin{aligned}
& \geq\left[\sum_{i=1}^{n} x_{i}^{\alpha_{n}} \operatorname{per} H_{n-1}(\mathbf{x}(j), \boldsymbol{\alpha}(n))\right]^{\frac{1}{|\boldsymbol{\alpha}|}}+\left[\sum_{i=1}^{n} y_{i}^{\alpha_{n}} \operatorname{per} H_{n-1}(\mathbf{y}(j), \boldsymbol{\alpha}(n))\right]^{\frac{1}{|\boldsymbol{\alpha}|}} \\
& =\left[H_{n}(\mathbf{x}, \boldsymbol{\alpha})\right]^{\frac{1}{\alpha \mid}}+\left[\operatorname{per} H_{n}(\mathbf{y}, \boldsymbol{\alpha})\right]^{\frac{1}{|\alpha|}} .
\end{aligned}
$$

That is to say, inequality (5) holds.

According to the theory of mathematical induction, inequality (5) is proved.

Next, note the continuity of both sides of (5) for the variable $\boldsymbol{\alpha}$. We know that inequality

(5) also holds for the case

$$
\boldsymbol{\alpha} \in[0,1]^{n}, \quad 0<|\boldsymbol{\alpha}| \leq 1
$$

From the above analysis we know that equality in (5) holds if $\mathbf{x}, \mathbf{y}$ are linearly dependent. This completes the proof of Theorem 1 .

\section{Applications in the theory of symmetric function}

We use the following notations in this section (see $[2,6,12,13])$ :

$$
\begin{aligned}
& \mathbb{N} \triangleq\{0,1,2, \ldots\}, \quad \mathscr{B}_{k}^{+} \triangleq\left\{\boldsymbol{\alpha} \in \mathbb{N}^{n}|| \boldsymbol{\alpha} \mid=k, k \in \mathbb{N}\right\}, \\
& \bar{P}_{k, n}[\mathbf{x}]=\left\{\sum_{\boldsymbol{\alpha} \in \mathscr{B}_{k}^{+}} \frac{\lambda(\boldsymbol{\alpha})}{n !} \operatorname{per} H_{n}(\mathbf{x}, \boldsymbol{\alpha}) \mid \lambda: \mathscr{B}_{k}^{+} \rightarrow(-\infty, \infty)\right\} \backslash\{0\}, \\
& \bar{P}_{k, n}^{+}[\mathbf{x}]=\left\{\sum_{\boldsymbol{\alpha} \in \mathscr{B}_{k}^{+}} \frac{\lambda(\boldsymbol{\alpha})}{n !} \operatorname{per} H_{n}(\mathbf{x}, \boldsymbol{\alpha}) \mid \lambda: \mathscr{B}_{k}^{+} \rightarrow[0, \infty)\right\} \backslash\{0\}, \\
& \sqrt[k]{\mathbf{x}} \triangleq\left(\sqrt[k]{x_{1}}, \ldots, \sqrt[k]{x_{n}}\right), \quad a-\mathbf{x}=\left(a-x_{1}, \ldots, a-x_{n}\right), \\
& G_{n}(\mathbf{x}) \triangleq \sqrt[n]{x_{1} \cdots x_{n}}, \quad \mathbf{I}_{n} \triangleq(1, \ldots, 1) \in(-\infty, \infty)^{n}, \\
& \mathbf{O}_{n} \triangleq(0, \ldots, 0) \in(-\infty, \infty)^{n},\left.\quad f_{1}^{\prime}\left(\mathbf{I}_{n}\right) \triangleq \frac{\partial f(\mathbf{x})}{\partial x_{1}}\right|_{\mathbf{x}=\mathbf{I}_{n}} .
\end{aligned}
$$

If $f(\mathbf{x}) \in \bar{P}_{k, n}[\mathbf{x}]$, then we call $f(\mathbf{x})$ a $k$-degree homogeneous and symmetric polynomial (see [6]). Obviously, we have that

$$
\bar{P}_{k, n}^{+}[\mathbf{x}] \subset \bar{P}_{k, n}[\mathbf{x}] .
$$

Theorem 1 implies the following result.

Theorem 2 Let $f(\mathbf{x}) \in \bar{P}_{k, n}^{+}[\mathbf{x}], k \geq 2$. Then, for any $\mathbf{x}, \mathbf{y} \in(0, \infty)^{n}$, we have the following Minkowski-type inequality:

$$
f(\sqrt[k]{\mathbf{x}+\mathbf{y}}) \geq f(\sqrt[k]{\mathbf{x}})+f(\sqrt[k]{\mathbf{y}})
$$

Equality in (45) holds if $\mathbf{x}, \mathbf{y}$ are linearly dependent.

Proof If $n=1$, then inequality (45) is an equation. We suppose that $n \geq 2$ below. 
Note that

$$
\frac{\boldsymbol{\alpha}}{k} \in[0,1]^{n}, \quad 0<\left|\frac{\boldsymbol{\alpha}}{k}\right|=1 \leq 1, \quad \forall \boldsymbol{\alpha} \in \mathcal{B}_{k}^{+} .
$$

According to Theorem 1, we get

$$
\begin{aligned}
f(\sqrt[k]{\mathbf{x}+\mathbf{y}}) & =\sum_{\boldsymbol{\alpha} \in \mathcal{B}_{k}^{+}} \frac{\lambda(\boldsymbol{\alpha})}{n !} \operatorname{per} H_{n}(\sqrt[k]{\mathbf{x}+\mathbf{y}}, \boldsymbol{\alpha}) \\
& =\sum_{\boldsymbol{\alpha} \in \mathcal{B}_{k}^{+}} \frac{\lambda(\boldsymbol{\alpha})}{n !}\left[\operatorname{per} H_{n}\left(\mathbf{x}+\mathbf{y}, \frac{\boldsymbol{\alpha}}{k}\right)\right]^{\frac{1}{\left|\frac{\alpha}{k}\right|}} \\
& \geq \sum_{\boldsymbol{\alpha} \in \mathcal{B}_{k}^{+}} \frac{\lambda(\boldsymbol{\alpha})}{n !}\left\{\left[\operatorname{per} H_{n}\left(\mathbf{x}, \frac{\boldsymbol{\alpha}}{k}\right)\right]^{\frac{1}{\left|\frac{\alpha}{k}\right|}}+\left[\operatorname{per} H_{n}\left(\mathbf{y}, \frac{\boldsymbol{\alpha}}{k}\right)\right]^{\frac{1}{\left|\frac{\alpha}{k}\right|}}\right\} \\
& =\sum_{\boldsymbol{\alpha} \in \mathcal{B}_{k}^{+}} \frac{\lambda(\boldsymbol{\alpha})}{n !}\left[\operatorname{per} H_{n}\left(\mathbf{x}, \frac{\boldsymbol{\alpha}}{k}\right)+\operatorname{per} H_{n}\left(\mathbf{y}, \frac{\boldsymbol{\alpha}}{k}\right)\right] \\
& =\sum_{\boldsymbol{\alpha} \in \mathcal{B}_{k}^{+}} \frac{\lambda(\boldsymbol{\alpha})}{n !} \operatorname{per} H_{n}(\sqrt[k]{\mathbf{x}}, \boldsymbol{\alpha})+\sum_{\boldsymbol{\alpha} \in \mathscr{B}_{k}^{+}} \frac{\lambda(\boldsymbol{\alpha})}{n !} \operatorname{per} H_{n}(\sqrt[k]{\mathbf{y}}, \boldsymbol{\alpha}) \\
& =f(\sqrt[k]{\mathbf{x}})+f(\sqrt[k]{\mathbf{y}}),
\end{aligned}
$$

that is to say, inequality (45) is proved. Equality in (45) holds if $\mathbf{x}, \mathbf{y}$ are linearly dependent by Theorem 1 .

The proof of Theorem 2 is completed.

Theorem 1 also contains the following result.

Theorem 3 Let $f:[0, a)^{n} \rightarrow[0, \infty)$ be a symmetric function, and $f(\mathbf{x})$ can be expressed as a convergent Taylor series:

$$
f(\mathbf{x})=\sum_{k=0}^{\infty} \sum_{\boldsymbol{\alpha} \in \mathcal{B}_{k}^{+}} \frac{\lambda(\boldsymbol{\alpha})}{n !} \operatorname{per} H_{n}(\mathbf{x}, \boldsymbol{\alpha}), \quad \forall \mathbf{x} \in[0, a)^{n}
$$

where

$$
a>1,\left.\quad \lambda(\boldsymbol{\alpha}) \triangleq \frac{1}{\alpha_{1} ! \alpha_{2} ! \cdots \alpha_{n} !} \frac{\partial^{k} f(\mathbf{x})}{\partial x_{1}^{\alpha_{1}} \partial x_{2}^{\alpha_{2}} \cdots \partial x_{n}^{\alpha_{n}}}\right|_{x=\mathbf{O}_{n}} \geq 0, \quad \forall k \in \mathbb{N}, \forall \boldsymbol{\alpha} \in \mathcal{B}_{k} .
$$

Then, for any $\mathbf{x}, \mathbf{y}, \mathbf{x}+\mathbf{y} \in(0, a)^{n}$, we have the following inequality:

$$
\left[\frac{f(\mathbf{x}+\mathbf{y})}{f\left(\mathbf{I}_{n}\right)}\right]^{\frac{f\left(\mathbf{I}_{n}\right)}{n f_{1}^{\prime}\left(\mathbf{I}_{n}\right)}} \geq G_{n}(\mathbf{x})+G_{n}(\mathbf{y})
$$

Equality in (47) holds if there is a real $\theta \in(0,1)$ such that

$$
\mathbf{x}=\theta \mathbf{I}_{n} \quad \text { and } \quad \mathbf{y}=(1-\theta) \mathbf{I}_{n}
$$

or $f(\mathbf{x})=x_{1} x_{2} \cdots x_{n}$ and $\mathbf{x}, \mathbf{y}$ are linearly dependent. 
Proof Obviously, we have that

$$
\sum_{k=0}^{\infty} \sum_{\boldsymbol{\alpha} \in \mathscr{B}_{k}^{+}} \lambda(\boldsymbol{\alpha})=f\left(\mathbf{I}_{n}\right) .
$$

Here we show that

$$
\sum_{k=0}^{\infty} \sum_{\boldsymbol{\alpha} \in \mathcal{B}_{k}^{+}}|\boldsymbol{\alpha}| \lambda(\boldsymbol{\alpha})=n f_{1}^{\prime}\left(\mathbf{I}_{n}\right)
$$

Note the following identities:

$$
\begin{aligned}
f_{1}^{\prime}(\mathbf{x}) & =\frac{\partial f(\mathbf{x})}{\partial x_{1}} \\
& =\frac{\partial}{\partial x_{1}} \sum_{k=0}^{\infty} \sum_{\boldsymbol{\alpha} \in B_{k}^{+}} \lambda(\boldsymbol{\alpha}) \frac{\operatorname{per} H_{n}(\mathbf{x}, \boldsymbol{\alpha})}{n !} \\
& =\sum_{k=0}^{\infty} \sum_{\boldsymbol{\alpha} \in \mathcal{B}_{k}^{+}} \frac{\lambda(\boldsymbol{\alpha})}{n !} \frac{\partial}{\partial x_{1}} \sum_{\sigma \in S_{n}} x_{1}^{\alpha_{\sigma(1)}} x_{2}^{\alpha_{\sigma(2)}} \cdots x_{n}^{\alpha_{\sigma(n)}} \\
& =\sum_{k=0}^{\infty} \sum_{\boldsymbol{\alpha} \in \mathcal{B}_{k}^{+}} \frac{\lambda(\boldsymbol{\alpha})}{n !} \sum_{\sigma \in S_{n}} \alpha_{\sigma(1)} x_{1}^{\alpha_{\sigma(1)}-1} x_{2}^{\alpha_{\sigma(2)}} \cdots x_{n}^{\alpha_{\sigma(n)}} .
\end{aligned}
$$

Hence

$$
\begin{aligned}
f_{1}^{\prime}\left(\mathbf{I}_{n}\right) & =\sum_{k=0}^{\infty} \sum_{\boldsymbol{\alpha} \in \mathcal{B}_{k}^{+}} \frac{\lambda(\boldsymbol{\alpha})}{n !} \sum_{\sigma \in S_{n}} \alpha_{\sigma(1)} \\
& =\sum_{k=0}^{\infty} \sum_{\boldsymbol{\alpha} \in \mathcal{B}_{k}^{+}} \frac{\lambda(\boldsymbol{\alpha})}{n !}(n-1) ! \sum_{\sigma(1)=1}^{n} \alpha_{\sigma(1)} \\
& =\sum_{k=0}^{\infty} \sum_{\boldsymbol{\alpha} \in \mathcal{B}_{k}^{+}} \frac{|\boldsymbol{\alpha}| \lambda(\boldsymbol{\alpha})}{n} \\
& =\frac{1}{n} \sum_{k=0}^{\infty} \sum_{\boldsymbol{\alpha} \in \mathcal{B}_{k}^{+}}|\boldsymbol{\alpha}| \lambda(\boldsymbol{\alpha}) .
\end{aligned}
$$

That is to say, equation (49) holds.

Set $\boldsymbol{\alpha}=\left(n^{-1}, n^{-1}, \ldots, n^{-1}\right)$ in Theorem 1 , we get

$$
G_{n}(\mathbf{x}+\mathbf{y}) \geq G_{n}(\mathbf{x})+G_{n}(\mathbf{y}), \quad \forall \mathbf{x}, \mathbf{y} \in(0, \infty)^{n}, \forall n \geq 1
$$

According to the A-G inequality (see [12]) or Hardy's inequality (1), we have

$$
\frac{\operatorname{per} H_{n}(\mathbf{x}, \boldsymbol{\alpha})}{n !} \geq\left[G_{n}(\mathbf{x})\right]^{|\boldsymbol{\alpha}|}, \quad \forall \mathbf{x} \in(0, \infty)^{n}
$$


Note the A-G inequality with weights:

$$
\sum_{i=1}^{\infty} \lambda_{i} x_{i} \geq|\lambda|\left(\prod_{i=1}^{\infty} x_{i}^{\lambda_{i}}\right)^{\frac{1}{|\lambda|}}, \quad \forall \mathbf{x}, \lambda \in(0, \infty)^{\infty}
$$

By (48)-(52), we get

$$
\begin{aligned}
f(\mathbf{x}+\mathbf{y}) & \geq \sum_{k=0}^{\infty} \sum_{\boldsymbol{\alpha} \in \mathscr{B}_{k}^{+}} \lambda(\boldsymbol{\alpha})\left[G_{n}(\mathbf{x}+\mathbf{y})\right]^{|\boldsymbol{\alpha}|} \\
& \geq \sum_{k=0}^{\infty} \sum_{\boldsymbol{\alpha} \in \mathscr{B}_{k}^{+}} \lambda(\boldsymbol{\alpha})\left[G_{n}(\mathbf{x})+G_{n}(\mathbf{y})\right]^{|\boldsymbol{\alpha}|} \\
& \geq f\left(\mathbf{I}_{n}\right)\left\{\prod_{k=0}^{\infty} \prod_{\boldsymbol{\alpha} \in \mathscr{B}_{k}^{+}}\left[G_{n}(\mathbf{x})+G_{n}(\mathbf{y})\right]^{|\boldsymbol{\alpha}| \lambda(\boldsymbol{\alpha})}\right\}^{\frac{1}{f\left(\mathbf{I}_{n}\right)}} \\
& =f\left(\mathbf{I}_{n}\right)\left[G_{n}(\mathbf{x})+G_{n}(\mathbf{y})\right]^{\frac{1}{f\left(\mathbf{I}_{n}\right)} \sum_{k=0}^{\infty} \sum_{\boldsymbol{\alpha} \in \mathscr{B}_{k}^{+}}|\boldsymbol{\alpha}| \lambda(\boldsymbol{\alpha})} \\
& =f\left(\mathbf{I}_{n}\right)\left[G_{n}(\mathbf{x})+G_{n}(\mathbf{y})\right]^{\frac{n f_{1}^{\prime}\left(\mathbf{I}_{n}\right)}{f\left(\mathbf{I}_{n}\right)}} .
\end{aligned}
$$

That is to say, inequality (47) holds.

According to the above analysis, we know that a sufficient condition of inequality (47) to be an equality is as follows: there is a real $\theta \in(0,1)$ such that

$$
\mathbf{x}=\theta \mathbf{I}_{n} \quad \text { and } \quad \mathbf{y}=(1-\theta) \mathbf{I}_{n}
$$

or $f(\mathbf{x})=x_{1} x_{2} \cdots x_{n}$ and $\mathbf{x}, \mathbf{y}$ are linearly dependent.

This completes the proof of Theorem 3 .

Theorem 3 implies the following result.

Corollary 1 Let $a \in(0, \infty), \mathbf{x}, \mathbf{y}, \mathbf{x}+\mathbf{y} \in(0,1+a)^{n}$. Then we have the following inequality:

$$
\left[G_{n}(1+a-\mathbf{x}-\mathbf{y})\right]^{a}\left[G_{n}(\mathbf{x})+G_{n}(\mathbf{y})\right] \leq a^{a} .
$$

Equality in (53) holds if there exists a real $\theta \in(0,1)$ such that

$$
\mathbf{x}=\theta \mathbf{I}_{n} \quad \text { and } \quad \mathbf{y}=(1-\theta) \mathbf{I}_{n} .
$$

Proof We construct an auxiliary function $f:[0,1+a)^{n} \rightarrow(0, \infty)$ as follows:

$$
\begin{aligned}
f(\mathbf{x}) & \triangleq \prod_{i=1}^{n} \frac{1}{1+a-x_{i}}=(1+a)^{-n} \prod_{i=1}^{n}\left(1-\frac{x_{i}}{1+a}\right)^{-1} \\
& =(1+a)^{-n} \prod_{i=1}^{n} \sum_{j=0}^{\infty}\left(\frac{x_{i}}{1+a}\right)^{j}
\end{aligned}
$$




$$
\begin{aligned}
& =(1+a)^{-n} \sum_{k=0}^{\infty} \sum_{\boldsymbol{\alpha} \in \mathcal{B}_{k}^{+}} \frac{\operatorname{per} H_{n}\left(\frac{\mathbf{x}}{1+a}, \boldsymbol{\alpha}\right)}{n !} \\
& =\sum_{k=0}^{\infty} \sum_{\boldsymbol{\alpha} \in \mathcal{B}_{k}^{+}}(1+a)^{-k-n} \frac{\operatorname{per} H_{n}(\mathbf{x}, \boldsymbol{\alpha})}{n !},
\end{aligned}
$$

i.e.,

$$
f(\mathbf{x})=\sum_{k=0}^{\infty} \sum_{\boldsymbol{\alpha} \in \mathcal{B}_{k}^{+}} \frac{\lambda(\boldsymbol{\alpha})}{n !} \operatorname{per} H_{n}(\mathbf{x}, \boldsymbol{\alpha}), \quad \forall \mathbf{x} \in[0,1+a)^{n}
$$

where

$$
\lambda(\boldsymbol{\alpha}) \equiv(1+a)^{-k-n}>0
$$

Then

$$
f\left(\mathbf{I}_{n}\right)=a^{-n}, \quad f_{1}^{\prime}\left(\mathbf{I}_{n}\right)=a^{-n-1}
$$

According to Theorem 3, for any $\mathbf{x}, \mathbf{y}, \mathbf{x}+\mathbf{y} \in(0,1+a)^{n}$, inequality (47) holds, i.e.,

$$
\left[a G_{n}\left(\frac{1}{1+a-\mathbf{x}-\mathbf{y}}\right)\right]^{a} \geq G_{n}(\mathbf{x})+G_{n}(\mathbf{y})
$$

that is to say, inequality (53) holds. Equality in (53) holds if there exists a real $\theta \in(0,1)$ such that

$$
\mathbf{x}=\theta \mathbf{I}_{n} \quad \text { and } \quad \mathbf{y}=(1-\theta) \mathbf{I}_{n}
$$

by Theorem 3 . This ends the proof.

Corollary 1 implies the following result.

Corollary 2 Let the functions $\varphi:[b, c] \rightarrow(0,1+a)$ and $\psi:[b, c] \rightarrow(0,1+a)$ be continuous, and let them satisfy the following conditions:

$$
a>0, \quad b<c, \quad \varphi(t)+\psi(t) \in(0,1+a), \quad \forall t \in[b, c] .
$$

Then we have the following inequality:

$$
\exp \left[a \frac{\int_{b}^{c} \log (1+a-\varphi-\psi)}{c-b}\right]\left[\exp \left(\frac{\int_{b}^{c} \log \varphi}{c-b}\right)+\exp \left(\frac{\int_{b}^{c} \log \psi}{c-b}\right)\right] \leq a^{a}
$$

Set $\mathbf{x} \in(0, \infty)^{|\varepsilon|}, f(\mathbf{x})=x_{1} x_{2} \cdots x_{|\varepsilon|}$ in Theorem 3. By

$$
\operatorname{det} H_{n}(\mathbf{x}, \boldsymbol{\varepsilon})=\prod_{1 \leq i<j \leq n}\left(x_{j}-x_{i}\right)
$$

(see [6]) and Theorem 3, we have the following Corollary 3. 
Corollary 3 Let $\mathbf{x}, \mathbf{y} \in(-\infty, \infty)^{n}$, and let

$$
x_{1}<x_{2}<\cdots<x_{n}, \quad y_{1}<y_{2}<\cdots<y_{n}, \quad n \geq 2 .
$$

Then we have the following Minkowski-type inequality:

$$
\sqrt[|\varepsilon|]{\operatorname{det} H_{n}(\mathbf{x}+\mathbf{y}, \boldsymbol{\varepsilon})} \geq \sqrt[|\varepsilon|]{\operatorname{det} H_{n}(\mathbf{x}, \boldsymbol{\varepsilon})}+\sqrt[|\varepsilon|]{\operatorname{det} H_{n}(\mathbf{y}, \boldsymbol{\varepsilon})}
$$

Equality in (56) holds if $\mathbf{x}, \mathbf{y}$ are linearly dependent.

Remark 1 If there exists a function $M(\mathbf{x})>0$ such that for any $\mathbf{x} \in[0, a)^{n}$, any nonnegative integer $k$ and any $\alpha \in \mathscr{B}_{k}$ we have

$$
\left|\frac{\partial^{k} f(\mathbf{x})}{\partial x_{1}^{\alpha_{1}} \partial x_{2}^{\alpha_{2}} \cdots \partial x_{n}^{\alpha_{n}}}\right| \leq M(\mathbf{x})
$$

then (46) holds and the Taylor series (46) converges (see [14]) by the theory of mathematical analysis.

Remark 2 The significance of Theorem 2 and Theorem 3 is to estimate the lower bounds of the increment of the symmetric functions

$$
f(\sqrt[k]{\mathbf{x}}) \text { and }\left[\frac{f(\mathbf{x})}{f\left(\mathbf{I}_{n}\right)}\right]^{\frac{f\left(\mathbf{I}_{n}\right)}{n f_{1}^{\prime}\left(\mathbf{I}_{n}\right)}},
$$

respectively.

\section{Competing interests}

The authors declare that they have no competing interests.

\section{Authors' contributions}

All authors contributed equally and significantly in this paper. All authors read and approved the final manuscript

\section{Author details}

${ }^{1}$ Institute of Mathematical Inequalities and Applications, Chengdu University, Chengdu, Sichuan 610106, P.R. China.

${ }^{2}$ Department of Mathematics and Computer Science, Longyan University, Longyan, Fujian 364012, P.R. China.

\section{Acknowledgements}

This work was supported in part by the Natural Science Foundation of China (No. 61309015) and in part by the Foundation of Scientific Research Project of Fujian Province Education Department of China (No. JK2012049).

Received: 1 November 2013 Accepted: 25 April 2014 Published: 13 May 2014

\section{References}

1. Wen, JJ, Gao, CB, Wang, WL: Inequalities of J-P-S-F type. J. Math. Inequal. 7(2), 213-225 (2013)

2. Wen, JJ, Wang, WL: Chebyshev type inequalities involving permanents and their applications. Linear Algebra Appl. 422(1), 295-303 (2007)

3. Wang, BY: An Introduction to the Theory of Majorizations. Beijing Normal University Press, Beijing (1990) (in Chinese)

4. Wen, JJ, Wang, WL: Inequalities involving generalized interpolation polynomial. Comput. Math. Appl. 56(4)، 1045-1058 (2008)

5. Wen, JJ, Cheng, SS: Closed balls for interpolating quasi-polynomials. Comput. Appl. Math. 30(3), 545-570 (2011)

6. Wen, JJ, Zhang, ZH: Jensen type inequalities involving homogeneous polynomials. J. Inequal. Appl. 2010, Article ID 850215 (2010)

7. Marshall, AW, Olkin, I: Inequalities: Theory of Majorization and Its Applications. Academic Press, New York (1979)

8. Gardner, RJ: The Brunn-Minkowski inequality. Bull. Am. Math. Soc. 39, 355-405 (2002)

9. Gardner, RJ: The Brunn-Minkowski inequality, Minkowski's first inequality, and their duals. J. Math. Anal. Appl. 245, 502-512(2000) 
10. Gao, CB, Wen, JJ: Theory of surround system and associated inequalities. Comput. Math. Appl. 63, 1621-1640 (2012)

11. Wen, JJ, Han, TY, Cheng, SS: Inequalities involving Dresher variance mean. J. Inequal. Appl. 2013, Article ID 366 (2013)

12. Wen, JJ, Cheng, SS, Gao, C: Optimal sublinear inequalities involving geometric and power means. Math. Bohem. 134(2), 133-149 (2009)

13. Pečarić, JE, Wen, JJ, Wang, WL, Lu, T: A generalization of Maclaurin's inequalities and its applications. Math. Inequal. Appl. 8(4), 583-598 (2005)

14. Wen, JJ, Han, TY, Gao, CB: Convergence tests on constant Dirichlet series. Comput. Math. Appl. 62(9), 3472-3489 (2011)

10.1186/1029-242X-2014-186

Cite this article as: Wen et al:. Minkowski-type inequalities involving Hardy function and symmetric functions. Journal of Inequalities and Applications 2014, 2014:186

Submit your manuscript to a SpringerOpen ${ }^{\odot}$ journal and benefit from:

- Convenient online submission

Rigorous peer review

Immediate publication on acceptance

- Open access: articles freely available online

- High visibility within the field

- Retaining the copyright to your article 